\title{
Targeted correction of point mutations in the low density lipoprotein receptor gene mediated by single-stranded oligonucleotides in vivo
}

\author{
FANG-LIN ZHANG ${ }^{1,2}$, WEI DONG ${ }^{3}$, XIAO-YAN WU ${ }^{1}$, SHU-QUN HU ${ }^{1}$, \\ YA-LIN XU ${ }^{1}$, WEI-WEI XU ${ }^{1}$, QI CHEN ${ }^{1}$ and LE-MING FAN ${ }^{1}$ \\ ${ }^{1}$ Atherosclerosis Research Center, Nanjing Medical University, Nanjing 210029, Jiangsu; \\ ${ }^{2}$ School of Medicine, Nanchang University, Nanchang 330006, Jiangxi; ${ }^{3}$ School of Chemical Engineering, \\ Nanjing University of Science and Technology, Nanjing 210094, Jiangsu, P.R. China
}

Received May 13, 2009; Accepted August 18, 2009

DOI: $10.3892 / \mathrm{mmr} 00000194$

\begin{abstract}
This study was designed to investigate the repair of point mutations in the low density lipoprotein receptor (LDLR) gene mediated by single-stranded oligonucleotides (SSOs) in vivo. Mutations in the LDLR gene are known to be the prime cause of familial hypercholesterolemia (FH). SSOs result in sequence-specific alterations leading to the correction of mutations. In the present study, the LDLR gene with a nonsense mutation (c660x) was fused to a luciferase reporter gene (p660-LDLR-luc) and introduced into mouse liver by hydrodynamic gene transfer. These mice were then injected via the tail vein with different SSOs complexed with polyethylenimine. Firefly luciferase activity present in hepatic cell lysate was measured to analyze repair efficiency. Restriction fragment length polymorphism analysis and direct sequencing were performed to affirm that the LDLR mutation was corrected. The results indicate that the LDLR mutation was corrected in the liver in vivo only in the presence of antisense SSOs (anti-SSOs). Our findings provide initial evidence that the point mutation in p660-LDLR-luc can be corrected by anti-SSO targeted repair in vivo. This may be a potential strategy for the treatment of $\mathrm{FH}$.
\end{abstract}

\section{Introduction}

More than $70 \%$ of circulating low density lipoproteins (LDLs) are removed from the blood via hepatic LDL receptor (LDLR)mediated endocytosis (1). Mutations in the LDLR gene are the primary cause of familial hypercholesterolemia $(\mathrm{FH})(2,3)$.

FH is characterized by high levels of serum LDL-cholesterol and advanced atherosclerosis, leading to coronary artery

Correspondence to: Dr Fang-Lin Zhang, Atherosclerosis Research Center, Nanjing Medical University, Nanjing 210029, P.R. China E-mail: zhangf105@gmail.com

Key words: familial hypercholesterolemia, mutation, gene therapy, gene expression disease (4). Pharmacotherapy, along with dietary modification, is generally helpful in patients with heterozygous $\mathrm{FH}$, but may not be adequate for those with homozygous FH. For the latter group, LDL-apheresis $(5,6)$ and liver transplantation $(7,8)$ are recommended. However, LDL-apheresis is severely compromised by its limited availability, high cost and difficulties involving vascular access, while liver transplantation involves considerable risk and requires lifelong immunosuppressive therapy. The limitations of current treatment options for $\mathrm{FH}$ have a negative impact on the morbidity and mortality rate of this disease. Therefore, the need to develop more efficient and less risk-prone strategies has become not only necessary but urgent.

Gene therapy has emerged as a promising alternative treatment option for FH. Based on the transplantation of autologous hepatocytes, liver-directed gene therapy with recombinant retroviruses carrying the LDLR gene has successfully countered LDLR deficiency in an animal FH model (9). However, this particular technique has a main drawback: hepatocytes isolated from surgically resected tissue are difficult to expand in culture. Furthermore, viral vectors result in long-term hepatotoxicity (10). Recently, it was reported that a $m d x^{5 \mathrm{cv}}$ point mutation mouse model with Duchenne muscular dystrophy was corrected in vivo by small single-stranded oligonucleotide (SSO) directed gene repair (11). The ideal strategy for treating genetic diseases is correcting DNA mutations at their endogenous loci without disrupting other genomic sequences. The advantage of gene repair is that the corrected gene can be maintained, expressed and regulated as a normal endogenous gene (12). A number of reports (13-16) show that SSOs containing a single mismatch to the target DNA cause a sequence-specific alteration in mammalian cells and yeast. These data suggest that site-directed gene repair may be a potential strategy for treating $\mathrm{FH}$, and prompted us to study the correction of point mutations in the LDLR gene employing a similar strategy in vivo.

To this end, we generated a mouse model in which LDLR cDNA carrying a nonsense mutation was fused to a firefly luciferase reporter gene and expressed specifically in the liver. Different SSOs complexed with polyethylenimine (PEI) (SSOs/ $\mathrm{PEI})$ were injected into the mice via the tail vein to correct the 


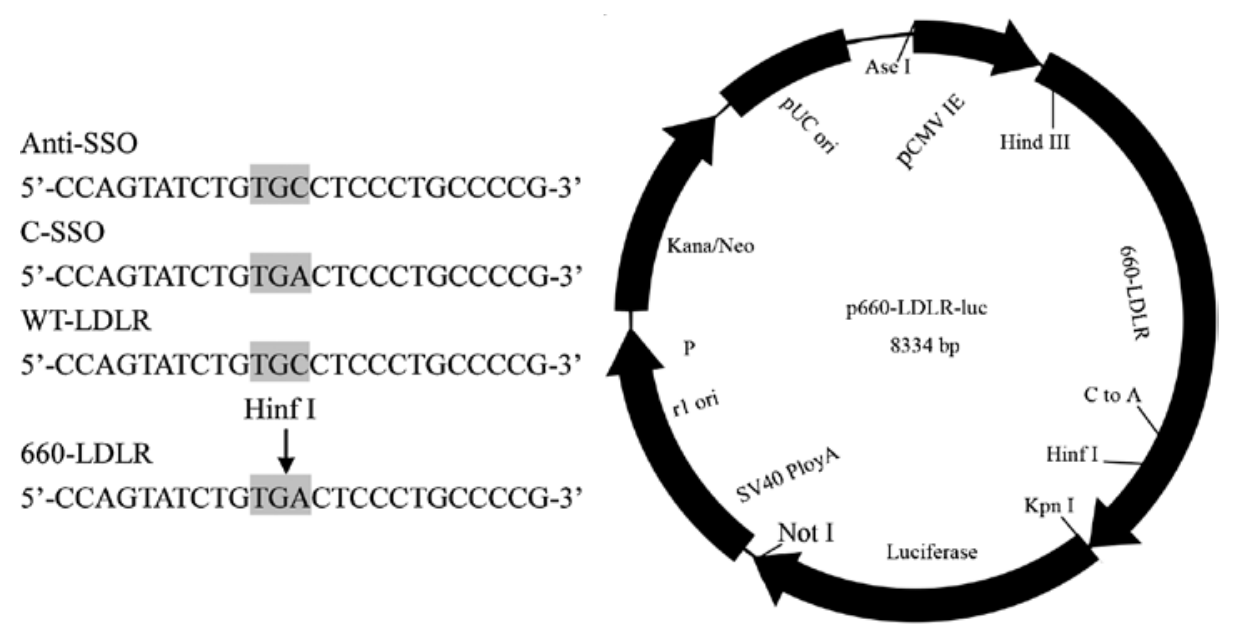

Figure 1. p660-LDLR-luc targeted gene correction system. p660-LDLR-luc contains a $\mathrm{C} \rightarrow \mathrm{A}$ transversion at nt 2726 , creating a premature termination codon and a HinfI site.

LDLR mutation. Our data suggest that antisense SSOs (antiSSOs) partially rescued the deficiency, thus providing novel evidence that anti-SSOs can be used for sequence-specific gene repair in the liver in vivo.

\section{Materials and methods}

Plasmid construction. The plasmids p660-LDLR-luc (660LDLR representing the LDLR gene with a nonsense mutation; luc representing the firefly luciferase gene) and pWT-LDLR-luc (WT-LDLR representing the wild-type LDLR gene) are derived from the plasmids p660-LDLR-EGFP (EGFP representing the enhanced green fluorescence protein gene) and pWT-LDLR-EGFP, respectively (kindly provided by Professor Thomas Gryesten Jensen of the Institute of Human Genetics, University of Aarhus), in which EGFP was replaced by luc p660-LDLR-luc carrying a $\mathrm{C} \rightarrow \mathrm{A}$ transversion, creating a premature termination codon and a HinfI site (Fig. 1). The validity of these plasmids was verified by restriction digestion with HinfI and direct sequencing.

Small single-stranded oligonucleotides. All SSOs (Invitrogen, Shanghai, P.R. China) were protected with four phosphorothioate linkages at the $5^{\prime}$ and $3^{\prime}$ ends, but fluorescein isothiocynate (FITC) SSOs (FITC-SSOs, the same sequence as anti-SSOs), were modified with 5' FITC and 3' phosphorothioate. Anti-SSOs targeting the non-transcribed strand contained a single mismatch to the target DNA, whereas the control SSOs (C-SSOs) contained no mismatch. The Anti-SSO and C-SSO sequences were 5'-CCAGTATCT GTGCCTCCCTGCCCCG-3' and 5'-CCAGTATCTGTGCCT CCCTGCCCCG-3', respectively.

Single-stranded oligonucleotide/polyethylenimine formation. Binary complex of GenEscort III (biodegradable hyperbranched PEI, Wisegen Biotechnology Corp., Nanjing, P.R. China) and SSOs were prepared (17) at a ratio of 6:1 PEI nitrogen/SSO phosphate $(\mathrm{N} / \mathrm{P}=6)$. SSOs and a corresponding amount of PEI solution were respectively diluted into $300 \mu \mathrm{l}$ phosphate buffered saline (PBS) and incubated for $10 \mathrm{~min}$ at room temperature. Subsequently, the PEI solution was added to the SSO solution, vortexed and incubated for $10 \mathrm{~min}$ at room temperature to allow complex formation. Immediately after incubation, SSOs/PEI were rapidly injected into the mice via the tail vein, avoiding mixing with the blood flow.

Animals. Male ICR mice (Shanghai Slac Laboratory Animal Co. Ltd., Shanghai, P.R. China) were 8-11 weeks of age with a body weight of 20-25 g when used for the experiments. Plasmids were diluted in sterile Ringer's solution $(50 \mu \mathrm{g} /$ ml). pWT-LDLR-luc, p660-LDLR-luc and pEGFP-N1 used as control solution $(1.6-2 \mathrm{ml})$ were injected into the mice via the tail vein by hydrodynamic gene transfer (HGT) (18-21). Subsequently, the mice transferred with p660-LDLR-luc were randomly assigned into anti-SSO, C-SSO and PEI groups $(n=6)$, and each group was injected with corresponding SSOs/ PEI $(1 \mu \mathrm{g} / \mathrm{g})$ via the tail vein $12 \mathrm{~h}$ after the transfer of the plasmids. The mice were sacrificed 3 days after the injection of the plasmids, and tissue samples were excised immediately. Experimental procedures were approved by the local legislation for ethics of experiments on animals.

Detection of plasmids delivered to mouse liver and sequence analysis. Liver tissue $(0.5 \mathrm{~g})$ was mixed with $1.0 \mathrm{ml}$ cell culture lysis reagent (Promega, Madison, WI, USA) and then incubated at $60^{\circ} \mathrm{C}$ for $5 \mathrm{~min}$, cooled to $40^{\circ} \mathrm{C}$ and incubated with $10 \mu \mathrm{l}$ of $10 \mathrm{mg} / \mathrm{ml}$ proteinase $\mathrm{K}$ for $2-3 \mathrm{~h}$. Subsequently, the plasmids present in lysis were extracted with EZNA plasmid Mini Kit I (Omega Bio-Tek Inc., Norcross, GA, USA). The plasmids were subjected to polymerase chain reaction (PCR) amplification with 5'-GGACAGATATCATCAACGAAG-3' and 5'-GCCTTTCTTTATGTTTTTGGCG-3' primers, generating an 830-bp fragment. For restriction fragment length polymorphism (RFLP) assay, PCR products were purified and digested with HinfI to confirm the correction of point mutations within the LDLR gene. Sequence analysis was performed by Invitrogen Co. (Shanghai, P.R. China).

Detection of fluorescein isothiocynate-single-stranded oligonucleotides in mouse liver. Mice were sacrificed $12 \mathrm{~h}$ after 


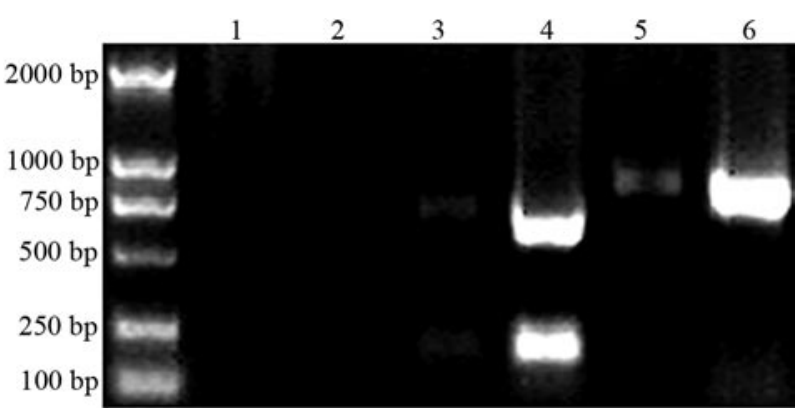

Figure 2. RFLP analysis of the 830-bp DNA fragments. Lanes 1, 3 and 5 are the HinfI digestion patterns of the PCR products generated from the mouse liver, transfected with control plasmid, p660-LDLR-luc and pWT-LDLR-luc, respectively. Lanes 2, 4 and 6 are the HinfI digestion patterns of the PCR products generated from control plasmid, p660-LDLR-luc and pWT-LDLR-luc, respectively.

the injection of FITC-SSOs/PEI (1 $\mu \mathrm{g} / \mathrm{g})$. PBS was used as the control. Frozen liver sections were examined by fluorescence microscopy (BX51 + DP70 + IPP, Olympus, Tokyo, Japan).

Luciferase assay. Tissues from mice were homogenized and lysed in cell culture lysis reagent (Promega). After a 15-min incubation at room temperature $(19,21)$ the lysed cells were collected and briefly centrifuged to remove cell debris. The firefly luciferase activity (FLA) present in $100 \mu$ l lysate was determined by the addition of $100 \mu \mathrm{l}$ of luciferase assay reagent (Promega). After $2 \mathrm{sec}$ of pre-incubation, the produced light was measured for $10 \mathrm{sec}$ in a TD-20/20 luminometer (Turner Designs, Sunnyvale, CA, USA). Protein concentrations were determined using the $\mathrm{BCA}^{\mathrm{TM}}$ protein assay kit (Pierce Co., OR, USA).

Statistical analysis. Data were analyzed for significant differences by the independent samples t-test. A p-value $<0.05$ was considered statistically significant. The tests were performed with SPSS version 10.0 for Windows (SPSS, Inc.). All values are presented as the mean \pm SD.

\section{Results}

LDLR-luc plasmids were delivered to the liver. Since the removal of LDL depends mainly on the hepatic LDL receptor, the liver was selected as the targeted organ for this study. HGT has been demonstrated as an efficient method of gene delivery to the liver $(18-20,22)$. Seventy two hours after the transfer of plasmids into mouse liver by HGT, we conducted PCR coupled with RFLP (PCR-RFLP) to determine whether the plasmids were delivered to mouse liver and whether the transfected p660-LDLR-luc were auto-corrected in vivo. An 830-bp fragment from mouse liver transfected with pWT-LDLR-luc or p660-LDLR-luc was amplified by a two-step PCR procedure. The DNA fragment was then digested with HinfI. As shown in Fig. 2, the DNA fragments from the p660-LDLR-luc group were completely digested by HinfI (lane 3), whereas these fragments from pWT-LDLR-luc group were not digested (lane 5). On the other hand, no amplification product was detected in the mouse liver transfected with control plasmids (pEGFP-N1) (lane 1). The results indicate that the plasmids were delivered to the mouse liver, and the point mutation in p660-LDLR-luc was not auto-corrected in the liver.

Expression of firefly luciferase in liver. Once the plasmids were delivered to the mouse liver, we examined the expression of pWT-LDLR-luc and p660-LDLR-luc in the liver. To this end, FLA present in hepatocyte lysates was assayed. As shown in Fig. 3A, the FLA of the pWT-LDLR-luc group $(n=6)$ reached $305.86 \pm 17.92$ light units/mg protein, whereas the FLA of the control plasmid group $(n=6)$ was only $0.75 \pm 0.3$ light units/ mg protein. However, in the p660-LDLR-luc group, low level expression of firefly luciferase was detected $(n=6 ; 21.7 \pm 1.59$ light units/mg protein), higher than that of the control plasmid group $(\mathrm{p}<0.001)$. The results suggest that the expression of luciferase was deficient but not $100 \%$ blunted by the nonsense mutation in LDLR.

Expression of firefly luciferase in various organs. To affirm that the plasmids were specifically delivered to the mouse liver, samples from other organs (heart, lung, spleen and kidney) of the mice transfected with pWT-LDLR-luc were collected for luciferase assay. As shown in Fig. 3B, FLA present in heart, lung, spleen and kidney lysates was drastically lower than that present in the liver $(\mathrm{p}<0.001)$, which clearly indicates that HGT resulted in a highly liver-specific gene transfer. Furthermore, assaying FLA present in hepatocyte lysates is an effective method for estimating the correction efficiency of the point mutation in p660-LDLR-luc.

Liver uptake of fluorescein isothiocyanate-single-stranded oligonucleotides. Since the point mutation in p660-LDLR-luc in mouse liver cannot be auto-corrected (as described above), the delivery of SSOs to mouse liver in vivo is a requirement for gene repair. Accordingly, FITC-SSOs/PEI or PBS (used as a control) was injected into the mouse tail vein. A fluorescence assay was performed $12 \mathrm{~h}$ after injection to determine whether SSOs/PEI were delivered to the mouse liver. As shown in Fig. 4, there was homogeneously diffused fluorescence in both sections, representing spontaneous fluorescence from the mouse liver. The vivid dots in Fig. 4B were generated by s-SSOs absorbed by the liver. These results indicate that FITC-SSOs/PEI were delivered to the mouse liver.

p660-LDLR-luc correction at the DNA level. To determine whether the point mutation in p660-LDLR-luc in liver in vivo was corrected by SSOs, we conducted both PCR-RFLP and DNA sequencing. As shown in Fig. 5A, the 830-bp DNA fragment amplified from the pWT-LDLR-luc group was not digested by HinfI (lane 2). Similarly, the same fragment amplified from the p660-LDLR-luc group followed by antiSSOs injection was resistant to HinfI digestion (lane 8; sample procured by two-step PCR, HinfI digestion, gel electrophoresis, recovery and purification, followed by two-step PCR and HinfI digestion). This indicates that the point mutation in p660-LDLR-luc was corrected. In contrast, fragments from the p660-LDLR-luc group injected with C-SSOs (lane 4) or PEI (lane 6) were digestable by HinfI, suggesting that the correction by anti-SSOs was specific. The 830 -bp DNA fragment from the corrected group was subjected to DNA sequencing. As shown in Fig. 5B, an $\mathrm{A} \rightarrow \mathrm{C}$ transversion at p660-LDLR-luc 
A

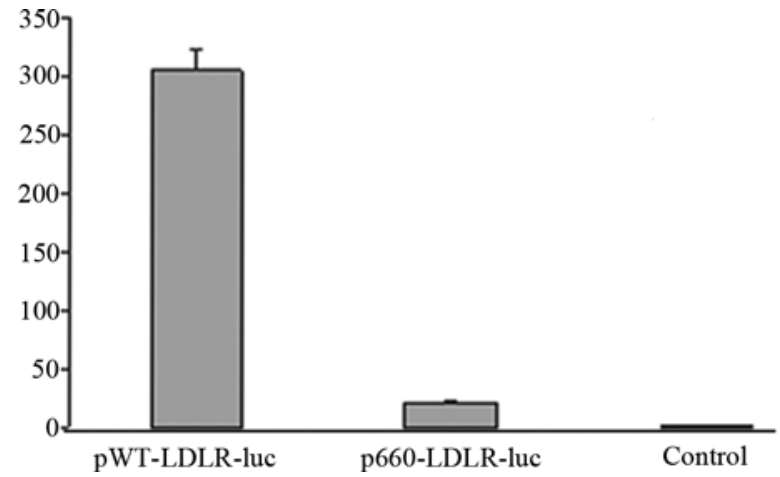

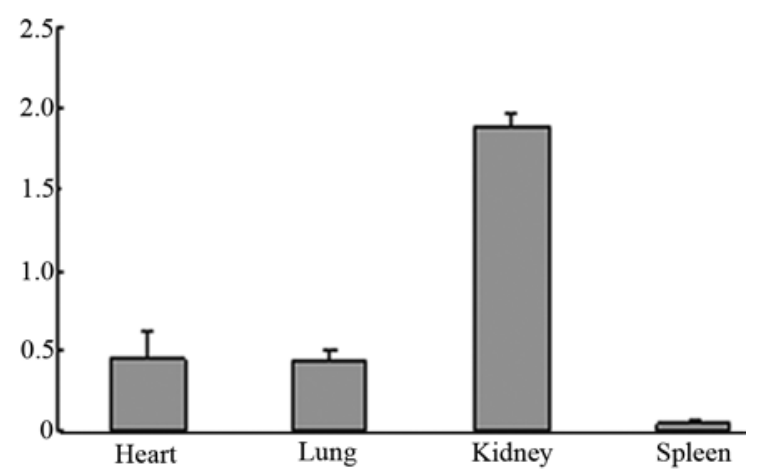

Figure 3. In vivo expression of firefly luciferase in the mice. (A) Firefly luciferase expressed in mouse liver transfected with pWT-LDLR-luc, p660-LDLR-luc and control plasmid, respectively. (B) Firefly luciferase expressed in the heart, lung, kidney and spleen of the mice transfected with pWT-LDLR-luc.

A

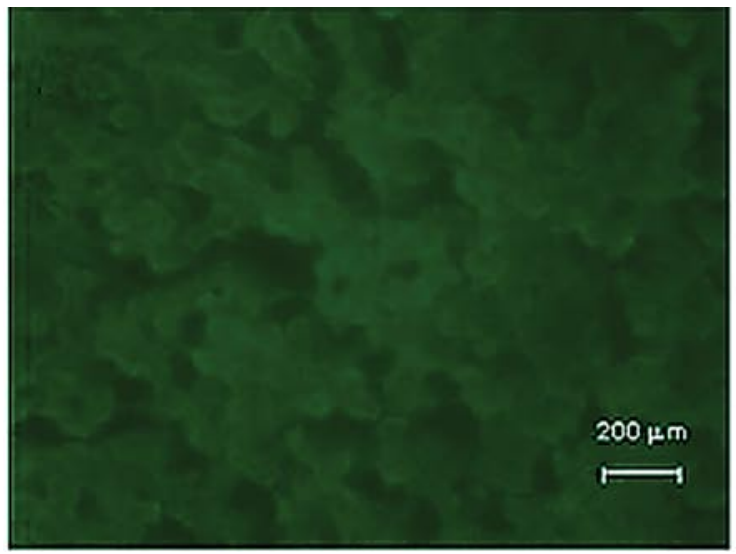

B

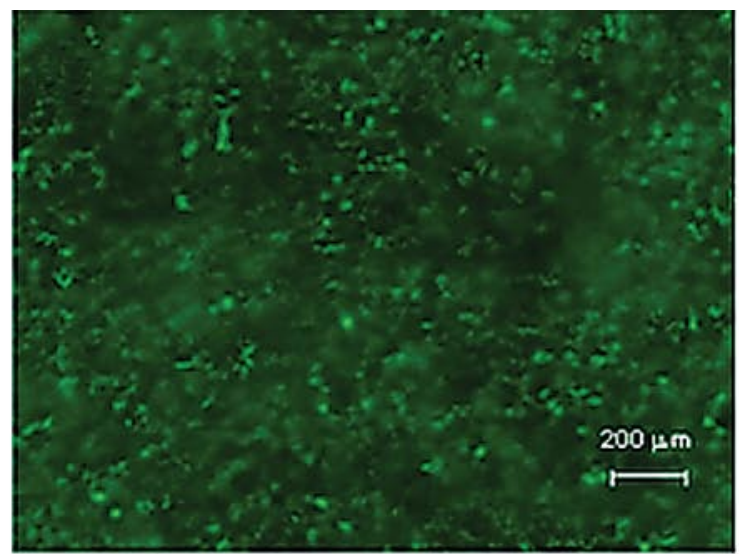

Figure 4. Liver uptake of FITC-SSOs. PBS and FITC-SSOs/PEI were injected into the mouse tail vein and fluorescence was observed $12 \mathrm{~h}$ after injection. (A) Frozen liver sections injected with PBS. (B) Frozen liver sections injected with FITC-SSOs/PEI (1 $\mu \mathrm{g} / \mathrm{g})$. The homogeneously diffused fluorescence in A and B is autochthonous fluorescence from the mouse liver. The vivid dots in B are FITC-SSOs absorbed by the mouse liver (x400; bar, $200 \mu \mathrm{m}$ ).

A

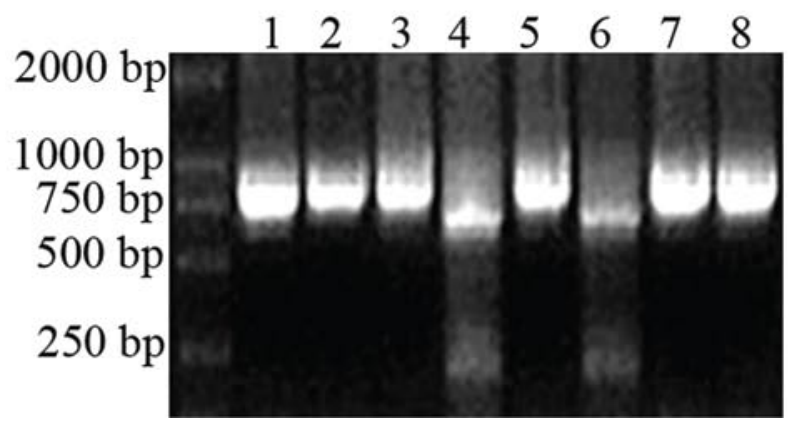

B

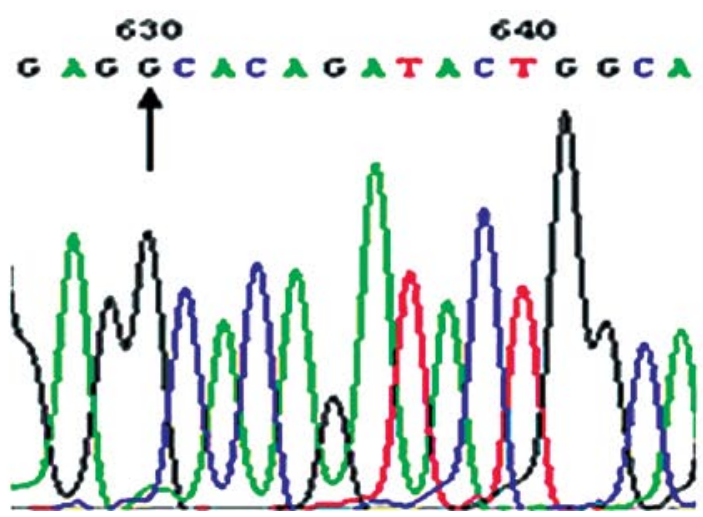

Figure 5. Evidence of p660-LDLR-luc correction at the DNA level. (A) RFLP analysis of the 830-bp DNA fragment. The fragments shown in lanes 1 and 2 , lanes 3 and 4, and lanes 5 and 6 were generated from the pWT-LDLR-luc, C-SSO and PEI groups respectively. The fragments shown in lanes 7 and 8 were generated from the anti-SSO group. Lanes 2, 4, 6 and 8 show HinfI digestion patterns, while in lanes 1, 3, 5 and 7 HinfI was undigested. The fragments shown in lanes 2, 4 and 6 were obtained by two-step PCR and HinfI digestion only. The sample in lane 8 was procured by two-step PCR, HinfI digestion, gel electrophoresis, recovery and purification, followed by two-step PCR and HinfI digestion. (B) DNA sequencing of the corrected DNA fragments. A was transfected into $\mathrm{C}$ in the LDLR gene.

was detected. Together, these data clearly indicate that the targeted gene was corrected at the DNA level in the presence of anti-SSOs alone.
p660-LDLR-luc correction at the protein level. To further affirm that the point mutation in p660-LDLR-luc in liver in vivo was corrected at the protein level, samples of mouse 


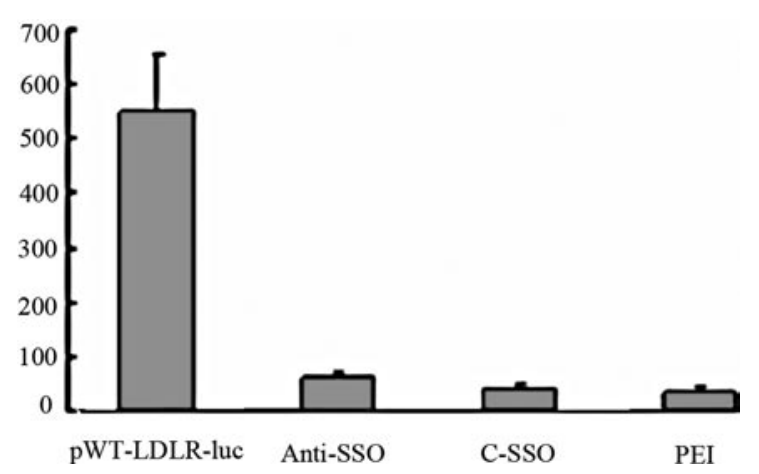

Figure 6. Expression of p660-LDLR-luc was elevated by anti-SSOs. Comparing the firefly luciferase activity (FLA) present in liver co-transfected with p660-LDLR-luc and anti-SSOs or C-SSOs showed that the FLA of the anti-SSO group was higher than that of C-SSO group $(\mathrm{p}<0.01)$.

liver treated with anti-SSOs, C-SSOs or PEI were collected for FLA assay. As shown in Fig. 6, the FLA of the anti-SSO group ( $\mathrm{n}=6 ; 60.51 \pm 10.26 \mathrm{light}$ units $/ \mathrm{mg}$ protein) was significantly higher than the FLA of the C-SSO group ( $n=6 ; 41.77 \pm 6.94$ light units/mg protein; $\mathrm{p}<0.005$ ) or the FLA of the PEI group $(\mathrm{n}=6 ; 32.55 \pm 9.66 ; \mathrm{p}<0.001)$, though there was no difference between C-SSOs and PEI ( $\mathrm{p}>0.05)$. However, the FLA of the anti-SSO group was significantly lower than the FLA of the pWT-LDLR-luc group $(n=6 ; 549.93 \pm 106.2$ light units/mg protein). Together, these results indicate that the mutation in p660-LDLR-luc was partially corrected by anti-SSOs.

\section{Discussion}

FH is recognized as one of the diseases for which gene therapy shows promising potential (23). However, there are significant shortcomings (e.g., toxicity, difficulty in expanding the primary hepatocyte in vitro) associated with gene augmentation strategy by retroviral vectors $(23,24)$. Our studies based on liver-specific gene repair strategy mediated by SSOs provide for the first time an alternative option for the treatment of FH homozygotes.

In the present study, the point mutation in p660-LDLR-luc was corrected in mouse liver in vivo by injecting anti-SSOs into mice via the tail vein. RFLP analysis, DNA sequencing and FLA assay showed that the correction occurred not only at the DNA level, but also at the protein level. It has also been reported that the $m d x^{5 \mathrm{cv}}$ point mutation mouse model with Duchenne muscular dystrophy was corrected in vivo by SSOs (11). These data suggest that site-directed gene repair in vivo is a potential strategy for treating genetic diseases.

The efficiency of mutation correction induced by SSOs depends on SSO delivery and release/access to the target site. Liver Kupffer and endothelial cells possess specific binding sites for SSOs (25). Free phosphorothioate SSOs are mainly distributed to the kidney and liver (26), while SSOs/PEI are mainly distributed to the liver and spleen (27). The complexation of SSOs with cationic lipids is not well suited for in vivo use (28). Therefore, we selected PEI as the delivery agent. In our study, a fluorescence assay clearly indicated that SSOs/PEI were present in mouse liver $12 \mathrm{~h}$ after intravenous injection. Robaczewska et al also demonstrated that SSOs/PEI were found in the liver $24 \mathrm{~h}$ after intravenous injection in four out of four injected ducks (17). These results indicate that PEI might be a potent system for the delivery of SSOs to mouse liver.

Reliable and sensitive assays play a critical role in the measurement of gene correction frequency. The advantage of the firefly luciferase assay is that it is rapid, sensitive and quantitative. In the present study, pWT-LDLR-luc and p660-LDLR-luc were constructed and transferred into mouse liver by HGT, in which the firefly luciferase gene was used as a reporter gene for the expression of LDLR. Our results clearly show that the plasmids were mainly delivered to the mouse liver and that the expression of the firefly luciferase gene was liver-specific. Expression of the firefly luciferase gene in p660-LDLR-luc was extremely low due to a nonsense mutation in the LDLR. However, expression of firefly luciferase was significantly elevated after the mice transfected with p660-LDLR-luc were treated with Anti-SSOs. The correction was further confirmed by RFLP analysis and DNA sequencing. These results suggest that the transfer of p660-LDLR-luc into mouse liver in vivo using HGT can be used as a method for verifying whether point mutation can be corrected in the liver in vivo by SSOs.

Two aspects of our results are noteworthy. First, in the p660-LDLR-luc group prior to Anti-SSOs treatment, a low level of FLA was detected (21.7 \pm 1.59 light units/mg protein); higher than that of the control plasmid group $(\mathrm{p}<0.001)$. However, RFLP analysis verified that p660-LDLR-luc was not corrected. This finding indicates that the expression of luciferase was deficient but not $100 \%$ blunted by the nonsense mutation in LDLR, which may be explained by the so-called 'read-through' of premature stop codon. Stop codon recognition is a slow process (29), and termination involves an active relationship between ribosome (rRNA and protein), mRNA, release factors and energy $(30,31)$. The termination process may be hindered by steric hindrance, thereby triggering readthrough. The mechanisms of the induction of read-through observed in this study require elucidation by further investigation. We also see the need to avoid overestimation when correction efficiency is estimated based only on the expression of downstream marker genes. The second aspect of the present study worth noting is the practicability of targeted gene repair strategy. The correction efficiencies reported so far are all far lower than those required for clinical application. There were also no visible improvements using our system, although we did not calculate the actual efficiency. Methods by which the efficiency of targeted gene repair may be substantially elevated are therefore the crucial objective for further studies.

In conclusion, a mouse model with a point mutated LDLR gene was successfully established by HGT, and SSOs carried by PEI corrected the point mutation in liver in vivo. The result is encouraging and further evaluation of this therapeutic strategy for the treatment of $\mathrm{FH}$ is warranted.

\section{Acknowledgements}

We thank Professor Thomas Gryesten Jensen of the Institute of Human Genetics, University of Aarhus for supplying the pWT-LDLR-EGFP and p660-LDLR-EGFP plasmids. We are also indebted to Dr Yong Xu and Dr Sheng-Nan Li from Nanjing Medical University for their inspiring input. This work was supported by the National Natural Science Foundation of China (Project no. 30470707). 


\section{References}

1. Garcia CK, Wilund K, Arca M, Zuliani G, Fellin R, Maioli M, Calandra S, Bertolini S, Cossu F, Grishin N, Barnes R, Cohen JC and Hobbs HH: Autosomal recessive hypercholesterolemia caused by mutations in a putative LDL receptor adaptor protein. Science 292: 1394-1398, 2001.

2. Soutar AK and Naoumova RP: Mechanisms of disease: genetic causes of familial hypercholesterolemia. Nat Clin Pract Cardiovasc Med 4: 214-225, 2007.

3. Tosi I, Toledo-Leiva P, Neuwirth C, Naoumova RP and Soutar AK: Genetic defects causing familial hypercholesterolaemia: identification of deletions and duplications in the LDL-receptor gene and summary of all mutations found in patients attending the Hammersmith Hospital Lipid Clinic. Atherosclerosis 194: 102-111, 2007.

4. Bujo H, Takahashi K, Saito Y, Maruyama T, Yamashita S, Matsuzawa Y, Ishibashi S, Shionoiri F, Yamada N and Kita T: Clinical features of familial hypercholesterolemia in Japan in a database from 1996-1998 by the research committee of the ministry of health, labour and welfare of Japan. J Atheroscler Thromb 11: 146-151, 2004.

5. Nakamura T and Ishikawa K: LDL-apheresis-clinical indication and utility. Nippon Rinsho 57: 2854-2861, 1999.

6. Thompson GR: LDL apheresis. Atherosclerosis 167: 1-13, 2003.

7. Alkofer BJ, Chiche L, Khayat A, Deshayes JP, Lepage A, Saloux E and Reznik Y: Liver transplant combined with heart transplant in severe heterozygous hypercholesterolemia: report of the first case and review of the literature. Transplant Proc 37: 2250-2252, 2005.

8. Khalifeh M, Faraj W, Heaton N, Rela M and Sharara AI: Successful living-related liver transplantation for familial hypercholesterolemia in the Middle East. Transpl Int 17: 735-739, 2005.

9. Chowdhury JR, Grossman M, Gupta S, Chowdhury NR, Baker JR Jr and Wilson JM: Long-term improvement of hypercholesterolemia after ex vivo gene therapy in LDLR-deficient rabbits. Science 254: 1802-1805, 1991.

10. Brunetti-Pierri N, Palmer DJ, Beaudet AL, Carey KD, Finegold M and $\mathrm{Ng}$ P: Acute toxicity after high-dose systemic injection of helper-dependent adenoviral vectors into nonhuman primates. Hum Gene Ther 15: 35-46, 2004.

11. Bertoni C, Morris GE and Rando TA: Strand bias in oligonucleotide-mediated dystrophin gene editing. Hum Mol Genet 14: 221-233, 2005.

12. Igoucheva O, Alexeev V and Yoon K: Oligonucleotide-directed mutagenesis and targeted gene correction: a mechanistic point of view. Curr Mol Med 4: 445-463, 2004.

13. Andrieu-Soler C, Halhal M, Boatright JH, Padove SA, Nickerson JM, Stodulkova E, Stewart RE, Ciavatta VT, Doat M, Jeanny JC, De Bizemont T, Sennlaub F, Courtois Y and BeharCohen F: Single-stranded oligonucleotide-mediated in vivo gene repair in the rd1 retina. Mol Vis 13: 692-706, 2007.

14. Igoucheva O, Alexeev V and Yoon K: Targeted gene correction by small single-stranded oligonucleotides in mammalian cells. Gene Ther 8: 391-399, 2001.

15. Yin W, Kren BT and Steer CJ: Site-specific base changes in the coding or promoter region of the human beta- and gamma-globin genes by single-stranded oligonucleotides. Biochem J 390: 253-261, 2005.
16. Yin WX, Wu XS, Liu G, Li ZH, Watt RM, Huang JD, Liu DP and Liang CC: Targeted correction of a chromosomal point mutation by modified single-stranded oligonucleotides in a GFP recovery system. Biochem Biophys Res Commun 334: 1032-1041, 2005.

17. RobaczewskaM, Guerret S, Remy JS, Chemin I, Offensperger WB, Chevallier M, Behr JP, Podhajska AJ, Blum HE, Trepo C and Cova L: Inhibition of hepadnaviral replication by polyethylenimine-based intravenous delivery of antisense phosphodiester oligodeoxynucleotides to the liver. Gene Ther 8: 874-881, 2001.

18. Budker VG, Subbotin VM, Budker T, Sebestyen MG, Zhang G and Wolff JA: Mechanism of plasmid delivery by hydrodynamic tail vein injection. II. Morphological studies. J Gene Med 8: 874-888, 2006.

19. He CX, Shi D, Wu WJ, Ding YF, Feng DM, Lu B, Chen HM, Yao JH, Shen Q, Lu DR and Xue JL: Insulin expression in livers of diabetic mice mediated by hydrodynamics-based administration. World J Gastroenterol 10: 567-572, 2004.

20. Wolff JA and Budker V: The mechanism of naked DNA uptake and expression. Adv Genet 54: 3-20, 2005.

21. Yeikilis R, Gal S, Kopeiko N, Paizi M, Pines M, Braet F and Spira G: Hydrodynamics based transfection in normal and fibrotic rats. World J Gastroenterol 12: 6149-6155, 2006.

22. Tada M, Hatano E, Taura K, Nitta T, Koizumi N, Ikai I and Shimahara Y: High volume hydrodynamic injection of plasmid DNA via the hepatic artery results in a high level of gene expression in rat hepatocellular carcinoma induced by diethylnitrosamine. J Gene Med 8: 1018-1026, 2006.

23. Marks D, Thorogood M, Neil HA and Humphries SE: A review on the diagnosis, natural history, and treatment of familial hypercholesterolaemia. Atherosclerosis 168: 1-14, 2003.

24. Sibley C and Stone NJ: Familial hypercholesterolemia: a challenge of diagnosis and therapy. Cleve Clin J Med 73: 57-64, 2006.

25. Biessen EA, Vietsch H, Kuiper J, Bijsterbosch MK and Berkel TJ: Liver uptake of phosphodiester oligodeoxynucleotides is mediated by scavenger receptors. Mol Pharmacol 53: 262-269, 1998.

26. Butler M, Crooke RM, Graham MJ, Lemonidis KM, Lougheed M, Murray SF, Witchell D, Steinbrecher $U$ and Bennett CF: Phosphorothioate oligodeoxynucleotides distribute similarly in class A scavenger receptor knockout and wild-type mice. J Pharmacol Exp Ther 292: 489-496, 2000.

27. Fischer D, Osburg B, Petersen H, Kissel T and Bickel U: Effect of poly(ethylene imine) molecular weight and pegylation on organ distribution and pharmacokinetics of polyplexes with oligodeoxynucleotides in mice. Drug Metab Dispos 32: 983-992, 2004.

28. Bijsterbosch MK, Manoharan M, Dorland R, van Veghel R, Biessen EA and Van Berkel TJ: bis-Cholesteryl-conjugated phosphorothioate oligodeoxynucleotides are highly selectively taken up by the liver. J Pharmacol Exp Ther 302: 619-626, 2002.

29. Curran JF and Yarus M: Use of tRNA suppressors to probe regulation of Escherichia coli release factor 2. J Mol Biol 203: 75-83, 1988.

30. Kisselev L, Ehrenberg M and Frolova L: Termination of translation: interplay of mRNA, rRNAs and release factors? EMBO J 22: 175-182, 2003.

31. Nakamura $\mathrm{Y}$ and Ito $\mathrm{K}$ : Making sense of mimic in translation termination. Trends Biochem Sci 28: 99-105, 2003. 